\title{
В.Я. ХРЫЩАНОВИЧ
}

\section{ПОСТТРОМБОТИЧЕСКАЯ БОЛЕЗНЬ: ДИАГНОСТИКА, ЛЕЧЕНИЕ, ПРОФИЛАКТИКА}

\author{
УО «Белорусский государственный медицинский университет», г. Минск, \\ Республика Беларусь
}

\begin{abstract}
Посттромботическая болезнь (ПТБ) оказывает серьезное негативное влияние на здоровье пациентов и наносит существенный экономический ущерб бюджету здравоохранения. Являясь следствием тромбоза глубоких вен (ТГВ), частичная или полная окклюзия вен, а также клапанная дисфункция, приводят к повышению венозного давления в микроциркуляторном сосудистом русле. Наряду с тем, что воспалительный процесс при ТГВ может способствовать рассасыванию тромбов, он также вызывает разрушение венозных клапанов. Диагностика ПТБ основана преимущественно на клинических проявлениях, к коим относятся ощущение тяжести и боль в ногах, отек, зуд, судороги, трофическая язва и липодерматосклероз. Среди нескольких клинических шкал или классификаций ПТБ наиболее предпочтительной является шкала Villalta. Факторами риска развития ПТБ являются проксимальный ТГВ и рецидив тромбоза, ожирение, варикозная болезнь, неадекватная антикоагулянтная терапия ТГВ. При установленном диагнозе, лечение ПТБ проводят по тем же принципам, что и хронической венозной недостаточности с использованием компрессионной терапии и возвышенного положения нижних конечностей. Недостаточно изучена роль веноактивных препаратов в профилактике и лечении ПТБ. Хирургическое лечение может применяться у весьма ограниченного контингента пациентов. Дальнейшие исследования должны определить роль тромболизиса и тромбэктомии, которые уже к настоящему времени показали свой терапевтический потенциал. Большее внимание следует уделять назначению и использованию компрессионного трикотажа у пациентов, перенесших ТГВ.
\end{abstract}

Ключевые слова: посттромботическая болезнь, тромбоз глубоких вен, консервативное лечение, оперативное лечение, профилактика

Post-thrombotic disease (PTD) influences negatively on patient health and have a major economic impact to health-care services. Being the sequence of deep venous thrombosis (DVT) due to partial or complete venous occlusion and valvular reux leading to increasing of venous pressure in the microcirculatory vascular bed.

The inflammatory process at DVT can contribute to the resorption of blood thrombus and it causes the destruction of venous valves. The diagnosis of PTD is principally based on clinical manifestations such as feelings of heaviness or pain in the leg, swelling, itch, convulsions, trophic ulcer and lipodermatosclerosis. Villalta scale is considered to be the most preferable among some clinical scales or classification of PTD. Risk factors for PTD development include a proximal DVT and recurrent thrombosis as well as obesity and varicose veins, non-adequate anticoagulation therapy of DVT. The treatment of PTD is usually performed according to the same principles as chronic venous insufficiency with compression therapy application and lower extremities elevation position. The role of vasoactive drugs in prevention and treatment of PTD has been studied insufficiently. Surgery can be applied only to very limited number of patients. Further studies are desperately needed to find out the role of thrombolysis and thrombectomy which by this time seem to be promising treatment. For patients with DVT more attention should be given to prescribing and application of compression hosiery.

Keywords: post-thrombotic disease, deep venous thrombosis, conservative treatment, surgical treatment, prevention

Novosti Khirurgii. 2013 May-Jun; Vol 21 (3): 120-128

Post-thrombotic disease: diagnostics, treatment, prevention

V.Ja. Khryshchanovich

\section{Введение}

Посттромботическая болезнь (ПТБ) относится к хроническим заболеваниям, для которой являются характерными симптомы и признаки, возникающие после перенесенного тромбоза глубоких вен (ТГВ) [1, 2]. Последствия венозного тромбоза варьируют от незначительных изменений кожи до трофических язв больших размеров. ПТБ является наиболее распространенным поздним осложнением ТГВ и развивается у 20-50\% пациен- тов, перенесших ТГВ нижних конечностей, при этом в 7-10\% случаев протекает в тяжелой форме с трофическими язвами [3, 4, 5]. ПТБ негативно сказывается на показателях здоровья и качества жизни пациентов [6], что подтверждают недавние проспективные исследования. Результаты анкетирования указали на худшее качество жизни у пациентов с ПТБ по сравнению пациентами того же возраста, страдающих артритом, хроническими заболеваниями легких, диабетом, в то время как качество жизни в случаях тяжелой формы 
ПТБ было сопоставимо с таковым при злокачественных опухолях, сердечной недостаточности и ишемической болезни сердца $[7,8]$. Необходимо отметить значительный социально-экономический ущерб, наносимый ТГВ и ПТБ бюджету здравоохранения и страны в целом. Проведенный в Швеции в течение 15 лет анализ наблюдений показал, что дополнительные долгосрочные расходы на лечение осложнений ПТБ составляют 75\% от стоимости лечения первичного ТГВ [9].

\section{Патофизиология}

Патофизиология ПТБ изучена недостаточно, но очевидно, что в ее основе лежит длительная венозная гипертензия [10]. Развивающиеся после ТГВ полная или частичная обструкция вены и несостоятельность клапанов, приводят к повышению венозного давления в микроциркуляторном русле. Кроме того, хроническая венозная гипертензия может быть причиной клапанной дисфункции перфорантных вен. Высокое давление в системе глубоких вен распространяется на подкожную капиллярную сеть и увеличивает эндотелиальную проницаемость, следствием чего является высвобождение крупных молекул в интерстициальное пространство и снижение оксигенации кожного покрова. В peзультате наблюдается замедление репаративных процессов при повреждениях кожи или развитие липодерматосклероза и спонтанных трофических язв [11].

В остром периоде ТГВ свежие тромботические массы вызывают окклюзию вены, после чего происходит реканализация просвета сосуда. Реканализация является сложным процессом, включающим в себя фибринолиз, организацию тромба и неоваскуляризацию [12]. В проспективном исследовании Е. RoumenKlappe et al. [13] у 93 пациентов с проксимальным и дистальным венозным тромбозом была изучена роль степени тяжести ТГВ, рефлюкса крови и затруднения венозного оттока, дисфункции мышечно-венозной помпы голени в развитии ПТБ. По данным авторов, частота встречаемости ПТБ через один год составила $46 \%$ и увеличивалась до 55\% и $56 \%$ через 2 и 6 лет соответственно. Наиболее важными прогностическими факторами развития ПТБ явились резидуальный тромбоз, рефлюкс и затруднение венозного оттока (обструкция или частичная реканализация), наблюдаемые через 3 месяца после выявления ТГВ. Полученные результаты позволили P. Prandoni и S. Kahn [14] предположить, что недостаточная реканализация в течение первых шести месяцев после эпизода венозного тромбоза может служить важным предиктором ПТБ.

Наряду с тем, что воспалительный процесс при ТГВ может способствовать лизированию тромбов, он также вызывает разрушение венозных клапанов, рефлюкс и последующее развитие ПТБ. В недавнем исследовании Н. Shbaklo et al. [15] была обнаружена убедительная взаимосвязь между воспалительными маркерами ИЛ-6, ICAM-1 и развитием ПТБ, что подтверждает существенное значение воспалительного процесса в патофизиологии ПТБ [16].

\section{Клинические проявления и диагностика}

Диагностика ПТБ основана преимущественно на клинических проявлениях, однако, у разных пациентов симптомы и признаки заболевания могут варьировать и носить неспецифический характер, что зачастую затрудняет постановку правильного диагноза. Наиболее характерными жалобами являются тяжесть и боль в ногах, отечность, зуд, судороги, язва и индурация кожи (таблица 1). Длительное стояние или ходьба, как правило, приводят к усилению симптомов и, наоборот, горизонтальное положение тела, возвышенное положение нижних конечностей и физический покой улучшают состояние. В редких

Типичные клинические проявления ПТБ

Таблица 1

\begin{tabular}{|c|c|}
\hline Симптомы (со стороны нижних конечностей) & Признаки \\
\hline $\begin{array}{l}\text { - тяжесть или слабость } \\
\text { - боль } \\
\text { • отечность } \\
\text { - зуд } \\
\text { • судороги } \\
\text { - парестезии } \\
\text { • распирающая боль } \\
\text { • усиление симптомов при физической активности, } \\
\text { длительном стоянии, ходьбе; облегчение симптомов во } \\
\text { время отдыха в горизонтальном положении }\end{array}$ & $\begin{array}{l}\text { • отек } \\
\text { • corona phlebectatica paraplantaris } \\
\text { • вторичный варикоз } \\
\text { • гиперпигментация } \\
\text { - гиперемия } \\
\text { • преходящий цианоз } \\
\text { - липодерматосклероз } \\
\text { • экзема } \\
\text { • зажившая или открытая язва }\end{array}$ \\
\hline
\end{tabular}


случаях у пациентов с окклюзионной формой ПТБ могут наблюдаться венозная хромота и распирающие боли в ноге во время ходьбы.

Появлению симптомов и признаков ПТБ предшествует перенесенный в анамнезе и объективно подтвержденный ТГВ. Умеренная боль и небольшой отек появляются через несколько месяцев после разрешения острой фазы ТГВ, в связи с чем диагноз ПТБ является правомочным только через 3 месяца с момента развития флеботромбоза [1]. В настоящее время отсутствует золотой стандарт функциональной, лабораторной и визуализирующей диагностики заболевания. Вместе с тем, методы визуализации (ультразвуковое ангиосканирование, рентгеновская и магнитно-резонансная венография, компьютерная томография) помогают верифицировать наличие венозной обструкции и/или рефлюкса. Но даже внутрисосудистое ультразвуковое исследование, позволяющее оценить морфологические изменения при обструкции подвздошной вены, не дает объективной информации о характере гемодинамических изменений.

Предложено несколько клинических шкал для классификации и оценки степени тяжести ПТБ [1]. Классификация L. Widmer [2], клиническая, этиологическая, анатомическая и патофизиологическая классификация СЕАР [17], клинический тест для оценки тяжести венозной патологии (VCSS) [18] были разработаны для всех пациентов с хронической венозной недостаточностью/заболеваниями вен и могут быть применимы при ПТБ. Шкалы D. Brandjes [19], J. Ginsberg [20, 21], S. Villalta [22] разрабатывались специально для диагностики ПТБ, но, не являясь адаптированными к каждой конкретной клинической ситуации, они не позволяют проводить сравнительные исследования. Заседание международного общества тромбоза и гемостаза, состоявшееся в 2008 г. в Вене, рекомендовало, как наиболее удобную и специфичную, шкалу S. Villalta для диагностики и оценки степени тяжести ПТБ после перенесенного ранее и документально подтвержденного ТГВ (таблица 2) [1].

Согласно шкале S. Villalta сумма баллов $>5 \pm$ венозная язва подтверждают диагноз ПТБ, а большее количество баллов характеризует степень тяжести заболевания (5-14 легкая/умеренная, $\geq 15$ - тяжелая).

\section{Факторы риска развития посттромботической болезни}

Пол. Популяционное исследование «случай-контроль», проведенное в Голландии на 1668 пациентах, выявило более высокий риск развития ПТБ у женщин, чем у мужчин с частотой $31 \%$ vs $17 \%$ (RR [1,5, 1,3-1,8]) [23]. Однако данные других исследований опровергают возможное влияние гендерного признака на возникновение ПТБ $[24,25]$. В результате проспективного обследования 406 пациентов, проведенного M. Stain et al. [25], мужской пол являлся слабым фактором риска ПТБ.

Ожирение. Взаимосвязь ожирения с частотой развития ПТБ $[23,25,26]$ была продемонстрирована в одном из исследований и составила $34 \%$ через один год после перенесенного ТГВ (при индексе массы тела $\geq 30$ кг/м² [OR, 6,08; 95\% CI, 1,75-21,14]). Избыточный вес приводит к повышению венозного давления и потенцирует рефлюкс уже в патологически измененных венах. Кроме того,

Шкала S. Villalta [22]

Таблица 2

\begin{tabular}{|c|c|c|c|c|}
\hline Симптомы/признаки & Отсутствие & Слабо выражен & $\begin{array}{c}\text { Умеренно } \\
\text { выражен }\end{array}$ & Сильно выражен \\
\hline Симптомы & & & & \\
\hline $\begin{array}{l}\text { Боль } \\
\text { Судороги } \\
\text { Чувство тяжести } \\
\text { Парестезии } \\
\text { Зуд } \\
\end{array}$ & $\begin{array}{l}0 \\
0 \\
0 \\
0 \\
0\end{array}$ & $\begin{array}{l}1 \\
1 \\
1 \\
1 \\
1 \\
\end{array}$ & $\begin{array}{l}2 \\
2 \\
2 \\
2 \\
2 \\
\end{array}$ & $\begin{array}{l}3 \\
3 \\
3 \\
3 \\
3 \\
\end{array}$ \\
\hline Признаки & & & & \\
\hline $\begin{array}{l}\text { Претибиальная гематома } \\
\text { Индурация кожи } \\
\text { Гиперпигментация } \\
\text { Гиперемия } \\
\text { Расширение вен } \\
\text { Болезненность при } \\
\text { компрессии голени } \\
\text { Венозная язва }\end{array}$ & $\begin{array}{l}0 \\
0 \\
0 \\
0 \\
0\end{array}$ & $\begin{array}{l}1 \\
1 \\
1 \\
1 \\
1 \\
1 \\
\text { Есть }\end{array}$ & $\begin{array}{l}2 \\
2 \\
2 \\
2 \\
2\end{array}$ & $\begin{array}{l}3 \\
3 \\
3 \\
3 \\
3 \\
3\end{array}$ \\
\hline
\end{tabular}


гиподинамия, наблюдаемая при ожирении, оказывает отрицательное влияние на работу мышечно-венозной помпы.

Варикозная болезнь в анамнезе еще до развития ТГВ также является фактором риска ПТБ [23]. У большинства пациентов с варикозом отмечается наличие рефлюкса, приводящего к повышению венозного давления и раннему развитию хронической венозной недостаточности.

Проксимальный тромбоз глубоких вен с распространением на подвздошно-бедренный сегмент и ипсилатеральный ТГВ в анамнезе служат строгими предикторами ПТБ [4, 23, $25,27]$. При проксимальном ТГВ вероятность развития ПТБ повышается в 2 раза по сравнению с дистальным ТГВ [25]. При этом локализация ТГВ (проксимальный или дистальный) не оказывала влияния на степень тяжести ПТБ ( $\mathrm{P}=0,109)$. По мнению некоторых авторов, рецидив венозного тромбоза также относится к факторам риска ПТБ [4, 28].

По предварительным данным повышенные уровни маркеров воспаления (ICAM-1, ИЛ-6, С-реактивный протеин, Р-селектин) и D-димера в самом начале ТГВ или в течение нескольких месяцев после установления диагноза свидетельствуют о риске развития ПТБ и рецидива венозного тромбоза [15].

Качество, интенсивность и длительность антикоагулянтной терапии. Ненадлежащие назначение и прием оральных антикоагулянтов при лечении острого венозного тромбоза повышали риск развития ПТБ [24]. В то же время, длительность антикоагулянтной терапии не оказывала влияния на частоту развития ПТБ [27]. По некоторым данным, в сравнении с антагонистами витамина К лучшая реканализация вен наблюдалась при длительном применении низкомолекулярного гепарина [24].

Возраст. Сведения о влиянии возраста на развитие ПТБ носят противоречивый характер. По данным L. Tick et al. [23] пациенты в возрасте старше 60 лет в меньшей степени были подвержены ПТБ, в то же время, в исследовании S. Kahn et al. [29] риск развития ПТБ был выше в старшей возрастной группе.
Продолжительность купирования симптомов тромбоза глубоких вен. При обследовании 387 пациентов S. Kahn et al. [29] было установлено, что неполное купирование симптомов, в первую очередь отека голени $(\geq 3$ см), в течение 1 месяца от начала развития ТГВ является строгим предиктором ПТБ в течение последующих двух лет.

Другие потенциальные факторы - злокачественные опухоли, хирургические вмешательства, незначительные повреждения, гипсовая иммобилизация, мутация V фактора Лейдена и протромбина 202104, не оказывали влияния на развитие ПТБ $[1,23,25,27,28$, 30].

В 2010 году P. Henke [31] была разработана прогностическая шкала SEAP для оценки риска развития ПТБ у пациентов с ТГВ (таблица 3), где $\mathrm{S}$ - степень тяжести симптомов ( 1 = отсутствие симптомов, 2 = незначительные симптомы, 3 = умеренные симптомы, 4 = выраженные симптомы, 5 = угроза потери ноги); Е - причины тромбоза (установлены или нет); А - анатомический сегмент (илиофеморальный, бедренно-подколенный, вены голени); Р - патофизиология (первичный, рецидивный). В настоящее время проводятся исследования, которые позволят адаптировать предложенную шкалу для использования в клинической практике.

\section{Лечение посттромботической болезни}

Консервативное лечение. При установленном диагнозе в основе лечения ПТБ лежат те же принципы, что и при хронической венозной недостаточности. Краеугольным камнем в лечении ПТБ является компрессионная терапия и возвышенное положение нижних конечностей, которые позволяют уменьшить отек, ускорить венозный отток и улучшить функцию мышечно-венозной помпы. Компрессионная терапия, как правило, является безопасным методом, но в случаях неправильного выбора степени компрессии может приводить к осложнениям. В настоящее время существует небольшое количество доказательств первого уровня в отношении эффек-

Шкала SEAP [31]

Таблица 3

\begin{tabular}{llll}
\hline \multicolumn{1}{c}{ Симптомы } & \multicolumn{1}{c}{ Этиология } & \multicolumn{1}{c}{ Анатомический сегмент } & Патофизиология \\
\hline $1=$ отсутствуют & $1=$ установлена & $1=$ вены голени & $1=$ первичный \\
$2=$ незначительные & $2=$ не установлена & $2=$ бедренно-подколенный & $2=$ рецидивный \\
$3=$ умеренные & & $3=$ илиофеморальный & \\
$4=$ выраженные & & $4=\geq 2$ сегментов & \\
$5=$ угрожающие & & & \\
\hline
\end{tabular}


тивности эластической компрессии при ПТБ [32]. Однако обоснованность ее применения была подтверждена многочисленными исследованиями у пациентов с хронической венозной недостаточностью [27].

В настоящее время наиболее приемлемой и лишенной целого ряда недостатков, присущих эластическим бинтам, является компрессионная терапия с использованием лечебного компрессионного трикотажа [31]. В основе его классификации лежит величина развиваемого давления на ткани нижней конечности, в зависимости от чего выделяют 4 класса компресии: I - 18-21 мм рт. ст., II - 23-32 мм рт.ст., III - 34-46 мм рт.ст., IV - более 49 мм рт.ст. При этом изделие обеспечивает физиологическое распределение давления: плавно снижается в проксимальном направлении, составляя в верхней трети голени - 70\% и на бедре $40 \%$ от максимального у лодыжек. В Республике Беларусь наибольшее распространение получили изделия "Sigvaris" (Швейцария), “Tonus Elast” (Латвия), "Venoteks" (США), которые выпускаются всех степеней компрессии и представлены большим ассортиментом размеров и моделей. Пациентам с ПТБ показано ношение лечебного трикотажа III компрессионного класса, при этом весьма важным моментом является необходимость регулярного его использования [32]. Выбор медицинского изделия (гольфы, чулки, колготы) зависит от локализации патологических изменений в венозной системе [27]: при дистальной локализации ПТБ необходимо использовать гольфы, при поражении проксимальных отделов - чулки, голготы. У большинства пациентов с ПТБ имеется распространенное поражение глубоких вен, поэтому им показано ношение чулок с застежкой на поясе.

J. Ginsberg et al. [33] было проведено рандомизированное перекрестное испытание на 15 пациентах прерывистой компрессии дважды в день в течение 2 месяцев, которое позволило улучшить симптомы ПТБ и уменьшить отек. В другом рандомизированном контролируемом исследовании на 26 пациентах с тяжелой формой ПТБ была изучена эффективность нового устройства для возврата венозной крови нижних конечностей (VENOPTS) [34]. Предварительные результаты свидетельствуют о возможности применения разработанного устройства в изолированном варианте или в комбинации с компрессионным трикотажем.

В основе фармакотерапии ПТБ лежит применение венотоников (уровень рекомендаций 2 А), среди которых наиболее изученным и часто используемым в Европе является ми- кронизированная очищенная флавоноидная фракция, представленная в Республике Беларусь под фирменным названием Детралекс ${ }^{\circledR}$. В жарких странах, где применение компрессионного лечения ограничено климатическими условиями, консервативные мероприятия, как правило, заключаются в монотерапии МОФФ, которая предотвращает разрушение протеогликанов капиллярной стенки, усиливает венозный тонус и скорость венозного оттока посредством противовоспалительного и вазоконстрикторного действия. В рандомизированном контролируемом исследовании A. Ramelet [35] показал эффективность МОФФ в купировании симптомов и признаков хронической венозной недостаточности, а в комбинации с эластической компрессией (уровень рекомендаций I A) и пентоксифиллином и сравнении с плацебо препарат оказывал позитивное влияние на процесс заживления трофической язвы [36]. В то же время, ощущается явный дефицит знаний об использовании венотоников у пациентов с ПТБ, не определены четкие показания, оптимальная дозировка, сроки лечения, безопасность приема этих препаратов в течение длительного периода времени. Несмотря на схожесть симптомов и признаков первичной и вторичной хронической венозной недостаточности, патофизиологические механизмы развития этих состояний различные. В дальнейшем необходимо проведение больших контролируемых исследований для оценки отдаленных результатов лечения веноактивными препаратами.

В настоящее время эффективность диуретиков и нестероидных противовоспалительных препаратов в купировании отека и других симптомов ПТБ не доказана [1]. В пяти рандомизированных контролируемых исследованиях была изучена роль пентоксифиллина (уровень рекомендаций 2 В) в заживлении трофической язвы в сочетании с эластической компрессией или без нее. Назначение препарата в дозировке 800 мг трижды в день привело к полной эпителизации язвы в течение 71 дня в отличие от плацебо контроля - 100 дней [37]. Одним из недостатков лечения пентоксифиллином являются желудочнокишечные расстройства, которые возникали у 10-20\% пациентов.

Хирургическое лечение применяется у весьма ограниченного контингента пациентов, отсутствуют четкие показания к выполнению хирургических вмешательств. Основанием к проведению последних могут быть тяжелые формы ПТБ, резистентные к консервативной терапии. При этом необходимо 
выяснить, чем обусловлена тяжесть проявлений заболевания - венозной обструкцией или рефлюксом. Традиционными хирургическими вмешательствами на уровне окклюзированного подвздошно-бедренного сегмента являются илио- или бедренно-кавальное и перекрестное бедренно-бедренное (операция Пальма) шунтирование. Реконструктивные операции на клапанах глубоких вен при ПТБ выполняются значительно реже, нежели у пациентов с первичной клапанной дисфункцией, однако в последние годы были получены обнадеживающие результаты при формировании неоклапанов [38, 39, 40]. Субфасциальная эндоскопическая диссекция перфорантных вен при ПТБ с серьезными трофическими нарушениями менее эффективна, чем у пациентов с первичной венозной недостаточностью (уровень рекомендаций II C) [41]. Тем не менее, у пациентов с тяжелыми проявлениями ПТБ удалось получить положительный эффект (купирование боли и отека, улучшение показателей качества жизни) после венозной ангиопластики \pm стентирования в $57 \%$ и $80 \%$ случаев соответственно [42].

\section{Профилактические мероприятия}

Стандартная терапия острого ТГВ заключается в назначении антикоагулянтов и эластической компрессии. Лечение антикоагулянтами предупреждает прогрессирование и рецидив ТГВ, легочную эмболию и летальный исход. Вместе с тем, оно не оказывает непосредственного влияния на процессы тромболизиса, который способствует ранней реканализации и сохранению венозных клапанов, что, в конечном счете, благоприятно сказывается на отдаленных результатах лечения [14]. Применение локального или катетер-направленного тромболизиса (ЛТ) позволяет в 50-70\% случаев восстановить просвет тромбированной вены [43], но отдаленные результаты и возможность предотвращения ПТБ остаются не изученными. Согласно обзору Cochrane, который включает 12 исследований, ЛТ снижает частоту развития ПТБ на $34 \%$ [44], а строгие критерии отбора пациентов могут улучшить безопас- ность и эффективность процедуры. Последнее руководство Американского колледжа пульмонологов (АССР) рекомендует выполнение ЛТ отобранным пациентам с распространенным проксимальным ТГВ, давность которого не более 14 дней, в учреждениях, имеющих достаточный опыт ЛТ и соответствующее оборудование (таблица 4). Кроме того, после проведения ЛТ может потребоваться баллонная ангиопластика и стентирование, а ожидаемая продолжительность жизни пациентов должна составлять 1 год и более. АССР не рекомендует выполнение чрескожной механической тромбэктомии, одобряет хирургическую тромбэктомию, но более предпочтительным считает чрескожный тромболизис [45].

Еще одним важным мероприятием, позволяющим предупредить развитие ПТБ, является профилактика рецидива венозного тромбоза, для реализации которой необходимо: 1) определить степень риска повторного ТГВ в зависимости от его распространенности, причин и связи с онкологическим заболеванием; 2) индивидуально для каждого пациента выбрать вид антикоагулянтной терапии, ее начало и продолжительность; 3) проводить антитромботическую профилактику у пациентов высокого медицинского и хирургического риска; 4) обучить пациентов и врачей различного профиля основным принципам лечения ТГВ и профилактике его рецидива. Некоторые авторы с этой целью предлагают наложение артерио-венозной фистулы после выполнения тромбэктомии из глубоких вен или ангиопластики/стентирования [43], но четкие критерии, регламентирующие показания к этой операции, к настоящему времени не разработаны. Кроме того, недостаточно изучены отдаленные результаты и клиническая эффективность прямой тромбэктомии из бедренно-подколенного сегмента.

Двумя рандомизированными контролируемыми исследованиями была показана эффективность эластического компрессионного трикотажа в снижении частоты развития ПТБ и его тяжести [19, 46, 47]. По данным D. Brandjes et al. [19], применение градуированной компрессии (30-40 мм рт. ст.) у пациентов с ТГВ в

Ранняя тромбэктомия и тромболизис при ТГВ [45]

Таблица 4

\begin{tabular}{lcc}
\hline \multicolumn{1}{c}{ Метод } & Доказательный уровень & Отсутствуют сведения \\
\hline Открытая тромбэктомия & I В & Кому показана? \\
Фармакологический ТЛ & II А & Когда начинать? \\
Фармако-механический ТЛ & II А & Скакого тромбированного \\
& & сегмента следует начинать? \\
Ангиопластика/стентирование & II В & - \\
\hline
\end{tabular}


течение 2 лет сократило частоту развития легких форм ПТБ с 47\% до 20\%, тяжелых форм ПТБ - с $23 \%$ до $11 \%$. В другом исследовании в результате использования компрессионного трикотажа развитие ПТБ было отмечено у 19 из 96 пациентов, в то время как, в группе сравнения ПТБ имел место у 44 из 90 волонтеров [46]. С другой стороны, небольшое исследование J. Ginsberg et al. [33] не выявило преимуществ эластической компрессии. Несмотря на то, что оптимальные сроки компрессионной терапии не установлены, АССР рекомендует пациентам с острым симптоматическим проксимальным ТГВ использовать градуированные компрессионные чулки или колготы с градиентом давления в области лодыжек 30-40 мм рт. ст. в течение, как минимум, двух лет (уровень рекомендаций I A) [45]. В противовес рекомендациям ACCP, M. Aschwanden et al. [48] было показано, что удлинение сроков стандартного компрессионного лечения более 6 месяцев не приносит дополнительных преимуществ. Очевидно, что необходимо проведение дальнейших исследований в этом направлении, однако достаточно трудно оценить все терапевтические эффекты эластической компрессии [46].

\section{Заключение}

ПТБ оказывает негативное влияние на здоровье и качество жизни пациентов. Компрессионная терапия до настоящего времени остается основным методом лечения большинства пациентов с ПТБ, несмотря на то, что в немалом количестве случаев возможно выполнение таких хирургических и рентгенологических вмешательств, как реконструкция клапанов или ангиопластика. Весьма перспективным направлением является изучение эффективности локальной тромболитической терапии и прямой тромбэктомии в сохранении функции венозных клапанов. В дальнейшем необходимо оценить возможности эндоваскулярных технологий при лечении «подострых» ТГВ (> 14 дней), а также разработать биомаркеры для определения давности тромбоза. Недостаточно изученной остается фармакотерапия ПТБ и, в частности, отдаленные результаты длительного применения веноактивных препаратов. В соответствии с рекомендациями Национального института здравоохранения Великобритании профилактика ТГВ и его рецидива - наиболее приемлемая превентивная стратегия, позволяющая сократить количество пациентов с ПТБ [49].

Конфликт интересов отсутствует

\section{ЛИТЕРАТУРА}

1. Definition of post-thrombotic syndrome of the leg for use in clinical investigations: a recommendation for standardization / S. R. Kahn [et al.] // J Thromb Haemost. - 2009 May. - Vol. 7, N 5. - P. 879-83.

2. Kolbach D. N. Definition of the post-thrombotic syndrome, differences between existing classifications / D. N. Kolbach, H. A. Neumann, M. H. Prins // Eur J Vasc Endovasc Surg. - 2005 Oct. - Vol. 30, N 4. - P. 404-14. 3. Kahn S. R. Frequency and determinants of the postthrombotic syndrome after venous thromboembolism / S. R. Kahn // Curr Opin Pulm Med. - 2006 Sep. Vol. 12, N 5. - P. 299-303.

4. The long-term clinical course of acute deep venous thrombosis / P. Prandoni [et al.] // Ann Intern Med. 1996 Jul 1. - Vol. 125, N 1. - P. 1-7.

5. Post-thrombotic syndrome, recurrence, and death 10 years after the first episode of venous thromboembolism treated with warfarin for 6 weeks or 6 months / S. Schulman [et al.] // J Thromb Haemost. - 2006 Apr. - Vol. 4, N 4. - P. 734-42.

6. Kahn S. R. Effect of postthrombotic syndrome on health-related quality of life after deep venous thrombosis / S. R. Kahn, A. Hirsch, I. Shrier // Arch Intern Med. - 2002 May 27. - Vol. 162, N 10. - P. 1144-48. 7. Post-thrombotic syndrome, functional disability and quality of life after upper extremity deep venous thrombosis in adults / S. R. Kahn [et al.] // Thromb Haemost. - 2005 Mar. - Vol. 93, N 3. - P. 499-502.

8. Determinants of health-related quality of life during the 2 years following deep vein thrombosis / S. R. Kahn [et al.] // J Thromb Haemost. - 2008 Jul. - Vol. 6, N 7. - P. 1105-12.

9. Cost of long-term complications of deep venous thrombosis of the lower extremities: an analysis of a defined patient population in Sweden / D. Bergqvist [et al.] // Ann Intern Med. - 1997 Mar. - Vol. 126, N 6. - P. 454-57.

10. Phillips L. J 2nd Molecular characterization of postthrombotic syndrome / L. J. 2nd Phillips, R. Sarkar // J Vasc Surg. - 2007 Jun. - Vol. 45. - Suppl A. - P. A116-22.

11. Singh H. Comparing short-term outcomes of femoral-popliteal and iliofemoral deep venous thrombosis: early lysis and development of reflux / H. Singh, E. M. Masuda // Ann Vasc Surg. - 2005 Jan. - Vol. 19, N 1. - P. 74-9.

12. Coagulation, fibrinolysis, and recanalization after acute deep venous thrombosis / M. H. Meissner [et al.] // J Vasc Surg. - 2002 Feb. - Vol. 35, N 2. - P. 278-85.

13. The post-thrombotic syndrome: incidence and prognostic value of non-invasive venous examinations in a six-year follow-up study / E. M. Roumen-Klappe [et al.] // Thromb Haemost. - 2005 Oct. - Vol. 94, N 4. - P. 825-30.

14. Prandoni P. Post-thrombotic syndrome: prevalence, prognostication and need for progress / P. Prandoni, S. R. Kahn // Br J Haematol. - 2009 May. - Vol. 145, N 3. - P. 286-95.

15. Shbaklo H. Levels of inflammatory markers and the 
development of the post-thrombotic syndrome / H. Shbaklo, C. A. Holcroft, S. R. Kahn // Thromb Haemost. - 2009 Mar. - Vol. 101, N 3. - P. 505-12.

16. Inflammation in deep vein thrombosis and the development of post-thrombotic syndrome: a prospective study / E. M. Roumen-Klappe [et al.] // J Thromb Haemost. - 2009 Apr. - Vol. 7, N 4. - P. 582-87. 17. Revision of the CEAP classification for chronic venous disorders: consensus statement / B. Eklöf [et al.] // J Vasc Surg. - 2004 Dec. - Vol. 40, N 6. - P. 1248-52. 18. Vasquez M. A. Venous Clinical Severity Score and quality-of-life assessment tools: application to vein practice / M. A. Vasquez, C. E. Munschauer // Phlebology. - 2008. - Vol. 23, N 6. - P. 259-75.

19. Randomised trial of effect of compression stockings in patients with symptomatic proximal-vein thrombosis / D. P. Brandjes [et al.] // Lancet. - 1997 Mar 15. Vol. 349, N 9054. - P. 759-62.

20. Postthrombotic syndrome after hip or knee arthroplasty: a crosssectional study / J. S. Ginsberg [et al.] // Arch Intern Med. - 2000 Mar 13. - Vol. 160, N 5. - P. 669-72.

21. Kahn S. R. Measurement properties of the Villalta scale to define and classify the severity of the postthrombotic syndrome / S. R. Kahn // J Thromb Haemost. - 2009 May. - Vol. 7, N 5. - P. 884-88.

22. Assessment of validity and reproducibility of a clinical scale for the post-thrombotic syndrome / S. Villalta [et al.] // Haemostasis. - 1994. - Vol. 24. -P. 158a. 23. Risk factors for post-thrombotic syndrome in patients with a first deep venous thrombosis / L. W. Tick [et al.] // J Thromb Haemost. - 2008 Dec. - Vol. 6, N 12. - P. 2075-81.

24. Relation between quality of anticoagulant treatment and the development of the postthrombotic syndrome / C. J. van Dongen [et al.] // J Thromb Haemost. - 2005 May. - Vol. 3, N 5. - P. 939-42.

25 . The post-thrombotic syndrome: risk factors and impact on the course of thrombotic disease / M. Stain [et al.] // J Thromb Haemost. - 2005 Dec. - Vol. 3, N 12. - P. 2671-76.

26. Body mass index is associated with the development of the post-thrombotic syndrome / W. Ageno [et al.] // Thromb Haemost. - 2003 Feb. - Vol. 89, N 2. - P. 305-9. 27. Kahn S. R. How I treat postthrombotic syndrome / S. R. Kahn // Blood. - 2009 Nov 19. - Vol. 114, N 21. - P. 4624-31.

28. Thrombophilia and the risk of post-thrombotic syndrome: retrospective cohort observation / L. Spiezia [et al.] // J Thromb Haemost. - 2010 Jan. - Vol. 8, N 1. - P. 211-13.

29. Determinants and time course of the postthrombotic syndrome after acute deep venous thrombosis / S. R. Kahn [et al.] // Ann Intern Med. - 2008 Nov 18. - Vol. 149, N 10. - P. 698-707.

30. Kahn S. R. Post-thrombotic syndrome after deep venous thrombosis: risk factors, prevention, and therapeutic options / S. R. Kahn // Clin Adv Hematol Oncol. - 2009 Jul. - Vol. 7, N 7. - P. 433-35.

31. Henke P. Prevention and treatment of the postthrombotic syndrome / P. Henke // J Vasc Surg. - 2010 Nov. - Vol. 52, N 5. - Suppl. - P. 21S-28S.
32. Compression therapy for treating stage I and II (Widmer) post-thrombotic syndrome / D. N. Kolbach [et al.] // Cochrane Database Syst Rev [Electronic resource]. - 2003. - N 4. - CD004177.

33. Intermittent compression units for severe post-phlebitic syndrome: a randomized crossover study / J. S. Ginsberg [et al.] // CMAJ. - 1999 May 4. - Vol. 160, N 9. - P. 1303-6.

34. Evaluation of a venous-return assist device to treat severe post-thrombotic syndrome (VENOPTS). A randomized controlled trial / M. J. O'Donnell [et al.] // Thromb Haemost. - 2008 Mar. - Vol. 99, N 3. - P. 623-29.

35. Ramelet A. A. Daflon 500 mg: symptoms and edema clinical update / A. A. Ramelet // Angiology. - 2005 Sep-Oct. - Vol. 56. - Suppl 1. - P. S25-32.

36. Secondary chronic venous disorders / M. H. Meissner [et al.] // J Vasc Surg. - 2007 Dec. - Vol. 46. Suppl S. - P. 68S-83S.

37. Systemic treatment of venous leg ulcers with high doses of pentoxifylline: efficacy in a randomized, placebo-controlled trial / V. Falanga [et al.] // Wound Repair Regen. - 1999 Jul-Aug. - Vol. 7, N 4. - P. 208-13.

38. Durability of venous valve reconstruction techniques for "primary" and postthrombotic reflux / S. Raju [et al.] // J Vasc Surg. - 1996 Feb. - Vol. 23, N 2. - P. - P. 357-66.

39. Maleti O Neovalve construction in postthrombotic syndrome / O. Maleti, M. Lugli // J Vasc Surg. - 2006 Apr. - Vol. 43, N 4. - P. 794-99.

40. Neovalve construction in deep venous incompetence / M. Lugli [et al.] // J Vasc Surg. - 2009 Jan. - Vol. 49, N 1. - P. 156-62, 162.e1-2.

41. Gloviczki P. Subfascial endoscopic perforator vein surgery: indications and results / P. Gloviczki // Vasc Med. - 1999. - Vol. 4, N 3. - P. 173-80.

42. Successful iliac vein and inferior vena cava stenting ameliorates venous claudication and improves venous outflow, calf muscle pump function, and clinical status in post-thrombotic syndrome / K. T. Delis [et al.] // Ann Surg. - 2007 Jan. - Vol. 245, N 1. - P. 130-9.

43. Catheter-directed thrombolysis and/or thrombectomy with selective endovascular stenting as alternatives to systemic anticoagulation for treatment of acute deep vein thrombosis / L. S. Jackson [et al.] // Am J Surg. 2005 Dec. - Vol. 190, N 6. - P. 864-68.

44. Watson L. I. Thrombolysis for acute deep vein thrombosis / L. I. Watson, M. P. Armon // Cochrane Database Syst Rev [Electronic resourse]. - 2004 Oct 18. - N 4. - CD002783.

45. American College of Chest Physicians. Antithrombotic therapy for venous thromboembolic disease: American College of Chest Physicians Evidence-Based Clinical Practice Guidelines (8th Edition) / C. Kearon [et al.] // Chest. - 2008 Jun. - Vol. 133, N 6. - Suppl. - P. 454S-45S

46. Below-knee elastic compression stockings to prevent the post-thrombotic syndrome: a randomized, controlled trial / P. Prandoni [et al.] // Ann Intern Med. - 2004 Aug 17. - Vol. 141, N 4. - P. 249-56.

47. Non-pharmaceutical measures for prevention of post-thrombotic syndrome / D. N. Kolbach [et al.] // 
Cochrane Database Syst Rev [Electronic resourse]. 2004. - N 1. - CD004174.

48. Effect of prolonged treatment with compression stockings to prevent post-thrombotic sequelae: a randomized controlled trial / M. Aschwanden [et al.] // J Vasc Surg. - 2008 May. - Vol. 47, N 5. - P. 1015-21. 49. Hill J. Reducing the risk of venous thromboembolism in patients admitted to hospital: summary of NICE guidance / J. Hill, T. Treasure // BMJ. - 2010 Jan 27. - Vol. 340. - P. 95.
Адрес для корреспонденции

220116, Республика Беларусь, г. Минск, пр-т Дзержинского, д. 83, УО «Белорусский государственный медицинский университет»,

2-я кафедра хирургических болезней, тел. раб: + 37517 287-86-52,

e-mail: vladimirkh77@mail.ru,

Хрыщанович Владимир Янович

\section{Сведения об авторах}

Хрыщанович В.Я., к.м.н., доцент 2-й кафедры хирургических болезней УО «Белорусский государ-

ственный медицинский университет».

\section{УВАЖАЕМЫЕ КОЛЛЕГИ!}

\section{3-26 октября 2013 года в г. Екатеринбург состоится РОССИЙСКИЙ НЕЙРОХИРУРГИЧЕСКИЙ ФОРУМ «НЕЙРООНКОЛОГИЯ» \\ ТЕМАТИКА ФОРУМА:}

Диагностика, хирургия и комплексное лечение опухолей центральной и периферической нервной систем.

1. Современные диагностические и лечебные технологии в нейроонкологии;

2. Глиальные и метастатические опухоли головного мозга;

3. Опухоли срединной линии и желудочковой системы;

4. Краниофациальные опухоли и опухоли основания черепа;

5. Другие опухоли головного мозга;

6. Опухоли позвоночника и спинного мозга;

7. Дисэмбриогенетические опухоли и объемные процессы;

8. Опухоли периферической нервной системы;

9. Нейроонкология в педиатрической нейрохирургии;

10. Комплексное лечение опухолей ЦНС: лучевые методики и химиотерапия;

11. Эндоваскулярные методы лечения.

Дополнительная информация на сайте: www.scaf-spb.ru 